\title{
The Application of Information Fusion in Reliability Evaluation of Complex Electromechanical System
}

\author{
Yongnan Wang ${ }^{1, \mathrm{a},}$ Xihui $\mathrm{Mu}^{2, \mathrm{~b}}$,Yueting $\mathrm{Niu}^{2, \mathrm{c}}$, Fengpo $\mathrm{Du}^{2, \mathrm{~d}}$, Jianhua Chen ${ }^{1, \mathrm{e}}$ \\ ${ }^{1}$ Mechanical Engineering College, Shijiazhuang 050003, China \\ ${ }^{2}$ Shijiazhuang New Technology Application Institute, Shijiazhuang 050003, China \\ a,b,c,d,e jxxywyn9999@163.com
}

Keywords: complex electromechanical system, reliability, information fusion technology.

\begin{abstract}
It is difficult to carry out the traditional reliability assessment test of large sample size; when evaluating system's reliability, due to high value and small samples.Mutual coupling of systen components.The difficulty to judge the impact and failure mode,The uncertainty of degraded performance parameters of that in the process of long-term storage. The information of the life characteristics contained in multi-source information.Therefore the method multi-source information fusion is needed.In view of the above problem, this paper summarizes the research status of information fusion technology,and the basic principle of data fusion,thingking,advantages and disadvantages as well as the integration mode, step and fusion step.
\end{abstract}

\section{The reliability evaluation status of complex electromechanical system}

Storage reliability refers to the products under specified conditions,within the prescribed storage time,maintain ability of stipulated by the state.It is one of the important indicators to measure product performance.Either long storage life or less maintenance in storage period is good to its storage reliability[1].With the new ideas putting forward and new technology researching,the integration automation and intelligent of products increased significantly,however,the rapid development also brought series of problems,especially the system reliability evaluation.At present, a set of evaluation methods has built up [2-4].Around the informatization offerings, however,no mature evaluation methods for products of high reliability, long life put forward.Problems in the process of reliability assessment include:First,generally the system is expensive,so the sample available to study is small and difficult to carry out large sample expereiment;Second, parallel system,serial system, redundant system involved, mutual coupling of systen components, the difficulty to judge the impact and failure mode,the uncertainty of degraded performance parameters of the system after long-term storage.The information of the life characteristics contained in multi-source information.All above,the study of evaluating reliability by the method multi-source information fusion.

\section{Information Fusion Technology}

Information fusion technology came from sensor technology of the military field,which has been widely applied to other areas,reliability evaluation is one of the branches.For some system reliability evaluation, because of the factors such as test conditions, funds etc,so other data sources had to be used, include the information such as (subsystem) system data, production data,the circuit testing results under natural environment, the data of the similar products and expert advice, etc[5].The problem above can be effectively solved by information fusion technology, with its wide coverage of space and time, powerful information extraction and comprehensive ability.

Basic Principle. The basic principle is the adoption of a certain fusion algorithm,dispose the reliability data collected in multiple channels, eliminate the error of the data and the contradictions between the data and get more accurate reliability evaluation result. Its object is reliability data, the core is coordinating and reliability information and comprehensive treatment[6].

The Basic Train of Thought. Usually information fusion includes two aspects:one is fusing all reliability information at the same life perid,the other is fusing reliability information of different 
life stages.The meanings of multi-source information fusion for complex system could be understood as two aspects: The data fusion at hierarchical level of reliability information about system and subsystem and even the smallest units at the same stage; fusing reliability information in the different life at all levels.

The Advantages and Disadvantages. Reliability information at various conditions of time and space can be made full use of so that the reliability information of the system more complete, and judgment information more accurate.At the same time, compared with classical reliability assessment methods, it is the use of spatio-temporal data from multiple sources that lead to the faults that large amount of information processing and step.

Integration Mode, Common Methods and Steps. The diversity of reliability information channel lead to the diversity of methods and fusion model. Common integration mode are as follows:

2.4.1 Fusion Model

(1)Parallel Fusion Model. As shown in the figure Fig. 1, generally applicable to reliability information from different sources while the type same, such as different expert experience information fusion;And the occasion the data needed have been obtained or data of equal status.

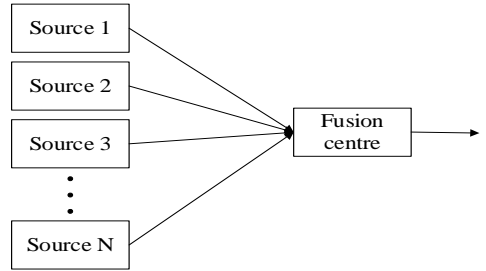

Fig. 1 Parallel Fusion Model

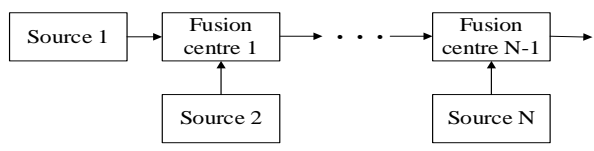

Fig. 2 Serial Fusion Model

(2)Serial Fusion Model. Serial fusion model is essentially a multistage parallel form of information fusion model between two sources. Generally it is available to certain occasions that various reliability information cellected by time. As shown in the figure Fig. 2.

(3) Mixed Fusion Model. Hybrid fusion model is the combination of methods above, which used more frequently.As evaluating the reliability of system,there are a lot of product development information, type test information and expert experience, similar product information and a small amount of field test information.In order to comprehensively utilizate all information, other reliability information expect field information usually, through the parallel fusion model, is used to build a final prior distribution, and then fuse the prior distribution and field data to get a posterior distribution, so as to deduce the reliability of products. Shown in the figure Fig. 3.

2.4.2 Main Method of Information Fusion and Analysis

(1)The Bayes Method. The Bayes method can improve the utilization efficiency of reliability information, particularly suitable for small sample, expensive test, but the influence of prior distribution on the result of the fusion is significant,and the way to make use of a priori information is an important problem in theory and engineering application[7]. As shown in the figure Fig. 4.

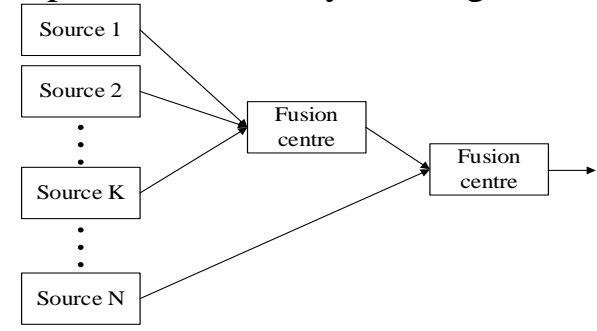

Fig. 3 Mixed Fusion Model

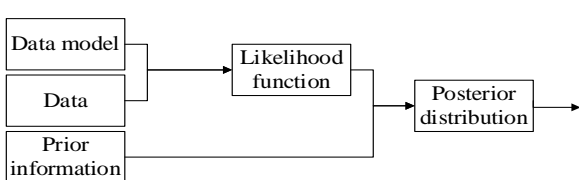

Fig. 4 The Bayes Method

(2) The Information Entropy Method. Information entropy is the expansion of the entropy theory, measure the information provide by the system, so that provide the basis of studying the theory of information fusion method, its advantages are closely integrated with the given information, while there is unified standard,which can only be based on the background of the specific issues and fusion target selection[7].

(3)The Fuzzy Integral Method. It is availible for expert experience information, unclear observation, reliability description of human, etc.make a quantitative analysis using the criterion so 
that the integral fuzzy integral value maximum. Its advantage lies in the real data to support possibility of the target appears match best with expectation.At the same time, membership function of the fuzzy concept is sensitive to the upper and lower bound,which limiting its ability as a general algorithm.the complexity of the fuzzy logic operation depends on the individual membership function structure,lacking a typical example to deal with by the logical implication of the membership function[7].

(4)D-S Evidence Reasoning Method. D-S evidence reasoning methods rely on evidence accumulation, narrowing assumptions set, make the shortest confidence interval of the fused. Advantage is that the mutual support between evidence and information fusion is more scientific and reasonable; Yet evidences shall be independent of each other, which limits its application[7].

2.4.3 Fusion Steps.Overall, reliability evaluation based on information fusion technology of system can be divided into three steps:

(1)Carding of the Reliability Information. Complete reliability information carding initially.Reliability information collected including product degradation performance parameters,data described as success or failure and descriptions of reliability by experts.These data's objects also are not identical.Such as system or subsystem information, and even information of parts.In addition,there is some abnormal data, such as "upside down" data and so on. Given these objects, methods and conditions of access to information, standards, etc. Each are not identical, and the data itself exists some exceptions.Therefore it's necessary to present reliability information before fusion.

(2)The Compatibility Test. The essence of compatibility test is testing whether the distribution obey same random totality.The study is aiming at deal with kinds of data,finding the extent of the data can be used.Checking the distribution of different data whether obey the same totality.If compatible,data can be fused. In addition, the compatibility test method research for different types of reliability information is rarely mentioned, in-depth studies are needed.

Common methods are Qualitative Analysis and Quantitative Analysis. Qualitative Analysis include Figure Comparasion Illustration and Time Series Model Comparasion,which is availible to simple comparasion of compatibility test problems in engineering research and simple test unit as well as the significant differences exit between samples. Qualitative Analysis is simple and fast but contentious. Quantitative Analysis is more accurate to test the compatibility between two samples, including dynamic consistency test and static consistency test.Dynamic consistency test is suitable for the observed value of random process, while static consistency test is suitable for the inspection of the observation value of the random variable.Assessing the reliability of system.Static consistency test include nonparametric test and parametric test. If the type of population distribution parameters are known,parametric test is relatively appropriate.If not, nonparametric test cab be used. Parametric test mainly includes the confidence intervals and hypothesis test. Nonparametric test including the Smilrove test, MOOD test, Rank sum test, etc[8].

(3)Reliability Information Fusion. After pretreatment and compatibility test, appropriate data fusion pattern and fusion method should be chosen to the data fusing process,life evaluation model will be concluded, residual life after storage of the product could be inferred.

\section{Conclusion}

With the study of information fusion technology explored successively,the information fusion technology could be perfected.High experiment expenses and little experiment data can be overcome,adopting appropriate fusion model and methed aiming at the actual situation. The information fusion technology beyond other metheds by its ability coverage of time and space.The hot research area of information fusion technology is the selection of fusion model and methed,and finding simplified algorithm on the basis of maintaining the accurate estimation.Along with the further research, information fusion technology will have a wider application prospect. 


\section{References}

[1] Huer Zhou.Researches on Bayesian Approach fou Storage Reliability Assessment of Ammunition System in Small Sample Circumstances and Design of its Application Sofeware[D]. Changsha: National University of Defense Technology, 2006.

[2] Bo Zheng, Baoqiang Yang, Bin Nan.The Estimation of Ammunition Storage Life in Field Environment[J]. Modern Defense Technology, 2003, (4).

[3] Gao Yulong, Jianzheng Yi, Haidan Wang,Analysis of Ammunition Technical Life Evaluation[J]. Journal of Environmental Engineering Equipment, 2011. (01).

[4] Zhifeng Fan, Ping Cui, Jing Lv .Estimation of Ammunition Control System Storage Life Based on Degradation Sensitive Parameters[J]. Journal of Arrows of the Guidance, 2013, (5).

[5] S Guibao ong, Fengwei Zhang.Research on the Method of Multistage Reliability Assessment Based on the Information Infusion Technology [J]. Journal of Ship Electrical Engineering, 2014, (02).

[6] Junge Fang.The Multi-source Information Fusion and its Application [J]. Journal of Gansu Science and Technology, 2013 (02).

[7] Huawei Wang. Analysis and Evaluation of Complex System Reliability [M]. Beijing: Science Press, 2013.

[8] Xiangping Zhang, Jinhuai Zhang etc. A Prior Information and the Sample of Compatibility Test Method Research [J]. Journal of Aircraft Measurement and Control, 2002, 21 (1) : 55-59 